\title{
Heterogeneity and restricted state selection in FRET with fluorescent proteins
}

\author{
T. S. Blacker ${ }^{1,2}$, M. R. Duchen ${ }^{2}$ and A. J. Bain ${ }^{1} *$ \\ 1. Department of Physics \& Astronomy, University College London, Gower Street, \\ London WC1E 6BT \\ 2. Department of Cell and Developmental Biology, University College London, \\ Gower Street, London WC1E 6BT
}

\begin{abstract}
Most fluorescent proteins exhibit multi-exponential fluorescence decays, indicating the presence of a heterogeneous excited state population. In the analysis of FRET to and between fluorescent proteins, it is often convenient to assume that a single interaction pathway is involved. However, in recent work we have shown that this assumption does not hold. Moreover, certain pathways can be highly constrained, leading to the potential misinterpretation of experimental data concerning protein-protein interactions. FRET and single-photon absorption both obey the same global electric dipole selection rules but differ greatly in the mechanism of the acceptor photoselection. In an isotropic medium, single-photon excitation accesses all acceptor transition dipole moment orientations with an equal probability. However, the FRET rate depends on the relative orientation of the donor and acceptor through the $\kappa^{2}$ orientation parameter. We show how time- and spectrally- resolved fluorescence intensity and anisotropy decay measurements following direct acceptor excitation, combined with those of the interacting FRET pair, can be used to identify restricted FRET state selection and thus provide accurate measurements of protein-protein interaction dynamics.
\end{abstract}

\section{KEYWORDS}

FRET, Time Resolved Fluorescence, Fluorescence Anisotropy, State Restriction, Fluorescent Proteins,

\section{BACKGROUND AND CONTEXT}

Förster resonance energy transfer (FRET) has become an important and widely used tool in the study of nanoscale molecular interactions, with applications spanning nanotechnology ${ }^{1}$, biophysics ${ }^{2}$ and the study of biochemical processes in live cells. The principles of FRET and its application as a nanometer ruler by Stryer and co-workers ${ }^{4}$ have been well established for over four decades with synthetic donor and acceptor fluorophores ${ }^{5}$. The development of genetically encodable fluorescent proteins as fluorescent markers ${ }^{6}$, and more recently as FRET donors and acceptors ${ }^{7}$, has been a particularly important milestone, as recognized by the 2008 Nobel Prize in Chemistry ${ }^{8}$. However, in contrast to synthetic fluorophores, fluorescent proteins regularly exhibit intrinsic heterogeneous excited state decay dynamics ${ }^{9-19}$ Recent work in our laboratory has shown that the donor-acceptor pathways in fluorescent protein FRET can be highly restricted and if unrecognized this can lead to significant errors in the interpretation of experimental data ${ }^{9}$. The impact of environmental heterogeneity on FRET when probing biological systems has also been recognized by a number of groups ${ }^{19,20}$. Intrinsic fluorescent probe and environmental heterogeneity therefore poses a significant problem for accurate quantitative FRET measurements.

*Corresponding Author, email: a.bain@ucl.ac.uk

Single Molecule Spectroscopy and Superresolution Imaging IX, edited by Jörg Enderlein, Ingo Gregor,

Zygmunt Karol Gryczynski, Rainer Erdmann, Felix Koberling, Proc. of SPIE Vol. 9714, 971401

(C) 2016 SPIE · CCC code: 1605-7422/16/\$18 · doi: 10.1117/12.2208895 


\section{RESTRICTED STATE SELECTION IN FRET BETWEEN EGFP \& MCHERRY}

\subsection{EGFP and mCherry Photophysics}

With both single and two-photon excitation, the fluorescence decay dynamics of non-protonated enhanced green fluorescent protein (EGFP) are well described by bi-exponential decays ${ }^{9-12}$, as summarized in Table 1. Fluorescence from EGFP therefore arises from two intrinsically different excited state populations. In contrast, the orientational dynamics of EGFP manifest in the fluorescence anisotropy is well described by a single rotational correlation time with an initial anisotropy close to the theoretical maximum for single or two-photon linearly polarized excitation ${ }^{9,17,18}$. The nature of the excited state heterogeneity in EGFP has yet to be determined. However, similar biexponential decay dynamics are observed in enhanced cyan fluorescent protein (ECFP) ${ }^{13,15}$, the shorter decay component being attributed to a conformation where the fluorescent chromophore experiences enhanced intramolecular quenching from His $148^{15}$. Recent X-ray crystallography work has pointed to a heterogeneous local environment for the EGFP chromophore arising from two distinct confirmations of Glu222 $2^{21}$.

\begin{tabular}{|c|l|l|l|l|l|l|c|}
\hline Sample & $\lambda_{\text {EXCITE }}$ & $\mathbf{A}_{\mathbf{1}}$ & $\begin{array}{l}\tau_{\mathbf{1}} \\
(\mathbf{n s})\end{array}$ & $\mathbf{A}_{\mathbf{2}}$ & $\begin{array}{l}\tau_{\mathbf{2}} \\
(\mathbf{n s})\end{array}$ & $\begin{array}{l}\langle\tau\rangle \\
(\mathbf{n s})\end{array}$ & $\boldsymbol{\lambda}_{\text {DET }} /$ Ref \\
\hline $\begin{array}{c}\text { EGFP-PDK1 } \\
\text { SEC pH 8.0 }\end{array}$ & $\begin{array}{c}2-\mathrm{P} \\
880 \mathrm{~nm}\end{array}$ & 0.503 & 3.067 & 0.497 & 2.43 & 2.75 & $\begin{array}{c}520 \mathrm{~nm}-542 \mathrm{~nm} \\
\text { Ref. 9 }\end{array}$ \\
\hline $\begin{array}{c}\text { EGFP-PDK1 } \\
\text { SEC pH 8.0 }\end{array}$ & $\begin{array}{c}1-\mathrm{P} \\
440 \mathrm{~nm}\end{array}$ & 0.622 & 3.001 & 0.378 & 2.304 & 2.738 & $\begin{array}{c}520 \mathrm{~nm}-542 \mathrm{~nm} \\
\text { Ref. } 10\end{array}$ \\
\hline $\begin{array}{c}\text { EGFP } \\
\text { SEC pH 8.0 }\end{array}$ & $\begin{array}{c}1-\mathrm{P} \\
440 \mathrm{~nm}\end{array}$ & 0.653 & 3.086 & 0.347 & 2.426 & 2.857 & $\begin{array}{c}630 \mathrm{~nm}-650 \mathrm{~nm} \\
\text { Ref. } 10\end{array}$ \\
\hline $\begin{array}{c}\text { EGFP } \\
\text { AB pH 7.2 }\end{array}$ & $\begin{array}{c}1-\mathrm{P} \\
485 \mathrm{~nm}\end{array}$ & 0.82 & 2.88 & 0.21 & 1.84 & 2.68 & $\begin{array}{c}510 \mathrm{~nm}-660 \mathrm{~nm} \\
\text { Ref. 11 }\end{array}$ \\
\hline $\begin{array}{c}\text { EGFP } \\
\text { AB pH 7.2 }\end{array}$ & $\begin{array}{c}2-\mathrm{P} \\
970 \mathrm{~nm}\end{array}$ & 0.80 & 2.90 & 0.21 & 1.85 & 2.68 & $\begin{array}{c}700 \mathrm{~nm}(\mathrm{SPF}) \\
\text { Ref. 11 }\end{array}$ \\
\hline $\begin{array}{c}\text { EGFP } \\
\text { AB pH 8.0 }\end{array}$ & $\begin{array}{c}1-\mathrm{P} \\
487 \mathrm{~nm}\end{array}$ & 0.86 & 2.63 & 0.14 & 1.36 & 2.45 & $\begin{array}{c}539 \mathrm{~nm}(\mathrm{IF}) \\
\text { Ref. 12 }\end{array}$ \\
\hline
\end{tabular}

Table 1: In vitro fluorescence decay measurements of EGFP were performed in aqueous media at $\mathrm{pH}$ values between 7 and 8, where the deprotonated form predominates. All measurements yield biexponential fluorescence decays characterized by a long component of c. 2.8-3.0 ns and a shorter minority decay component of ca. $1.8-2.0$ ns. All measurements yield a similar average fluorescence lifetime of ca $2.7 \mathrm{~ns}-2.8 \mathrm{~ns}$ with the exception of reference 12 where a maximum entropy method was applied to extract the decay components. Abbreviations: SEC $\equiv$ size exclusion chromatography buffer, $\mathrm{AB} \equiv$ aqueous buffer, EGFP-PDK1 $\equiv 3$-Phosphoinosotide dependent protein kinase 1 -EGFP, IF $\equiv$ interference filter, $\mathrm{SPF} \equiv$ short pass filter. 
One of the most widely used FRET acceptors for an EGFP donor is mCherry, with the strong overlap between donor emission and acceptor absorption giving rise to a calculated Förster radius of $5.24 \pm$ $0.10 \mathrm{~nm}^{22}$. FRET measurements between EGFP and mCherry in tandem constructs (where a high interacting fraction is expected) have indicated substantially lower values, the cause of which has been suggested to arise from incomplete maturation of mCherry, yielding a FRET inactive form ${ }^{11,23}$. Fluorescence from mCherry is characterized by a considerably lower quantum yield than EGFP $(0.22$ vs. 0.6 ) and a significantly shorter average lifetime (ca. 1.47 vs. $\left.2.75 \mathrm{~ns}^{9}\right)$. The reduced quantum yield of mCherry has been attributed to faster non-radiative relaxation arising from non-planarity of its chromophore $^{24}$. Formally, FRET is described in terms of simultaneous electric dipole allowed isoenergetic donor and acceptor de-excitation and excitation events mediated by the exchange of virtual photons $^{25}$. As a result, the spectrum of FRET excitations directly mirrors the overlap between the donor emission and acceptor absorption. It should be noted that this is maximized at energies greater than that of the single-photon absorption maximum of the FRET acceptor. In recent work ${ }^{9}$ we examined the fluorescence decay of mCherry with single-photon excitation wavelengths spanning the donor acceptor overlap region $(470 \mathrm{~nm}-610 \mathrm{~nm})$ as shown in Figure 1. Across this region, the fluorescence decay of mCherry was clearly bi-exponential, yielding a sub-1.6 ns fast (majority) component and a longer lived component of ca $3 \mathrm{~ns}$ in the blue which reduced with longer excitation wavelengths to a value of ca.1.8 ns at $610 \mathrm{~nm}$. The measured fluorescence lifetimes and their relative amplitudes are shown in figure 2 . Here it can be seen that a reduction in the weighting of the fast component is mirrored by a reduction in the lifetime of the slower component, with the effect that the average fluorescence lifetime showed only a slight decrease from ca $1.7 \mathrm{~ns}$ to $1.6 \mathrm{~ns}$ across the overlap region. If optical excitation and dipole-dipole transfer were equivalent then an overlap-weighted average of the excited state decays accessed by single-photon excitation of mCherry across this window should yield the same fluorescence decay as that for unrestricted FRET to mCherry. This average corresponded to two decay components with lifetimes of $1.47 \mathrm{~ns}$ and $2.77 \mathrm{~ns}$ with relative amplitudes of 0.844 and 0.156 respectively, corresponding to an average decay time of ca $1.67 \mathrm{~ns}$.

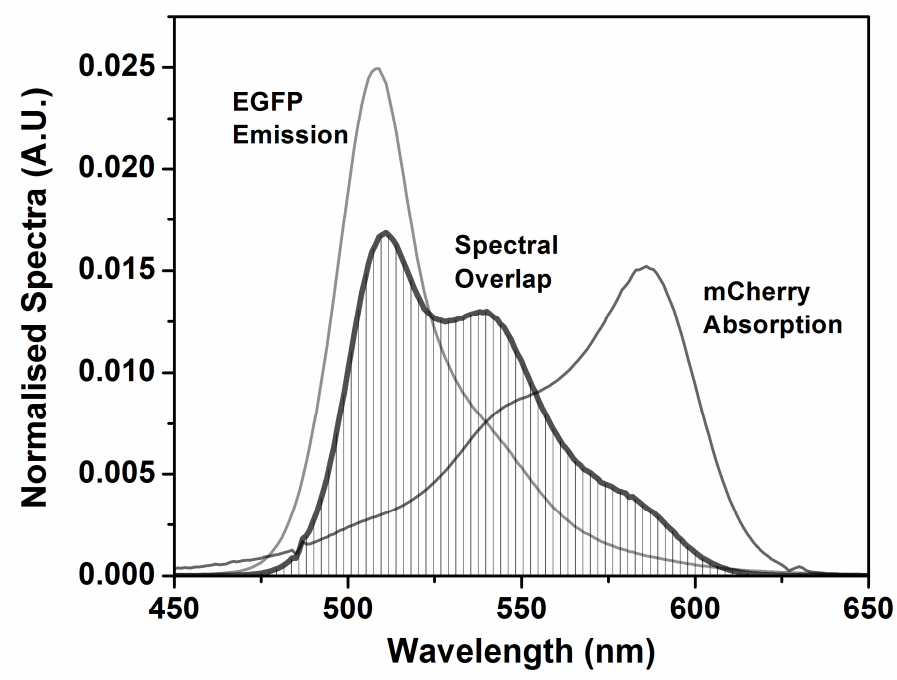

Figure 1: EGFP emission and mCherry absorption spectral overlap; FRET between EGFP and mCherry corresponds to single-photon excitation of mCherry at predominantly shorter wavelengths than the absorption maximum of mCherry. Reprinted with permission from "Restricted State Selection in Fluorescent Protein Förster Resonance Energy Transfer", Thomas A. Masters, Richard J. Marsh, Daven A. Armoogum, Nick Nicolaou, Banafshé Larijani, and Angus J. Bain, Journal of the American Chemical Society 2013135 (21), 7883-7890 DOI: 10.1021/ja312230b Copyright 2013 American Chemical Society. 
In our recent study of FRET between EGFP and mCherry, we employed the in vitro 3-Phosphoinositide Dependent Protein Kinase 1 (PDK1) dimerization system ${ }^{10}$ to study state selection using time-resolved intensity and anisotropy measurements. To characterize the heterogeneous populations involved in energy transfer, fluorescence intensity decays were recorded in two spectral windows spanning $520 \mathrm{~nm}$ to $542 \mathrm{~nm}$ dominated by EGFP emission (the donor window) and $630 \mathrm{~nm}$ to $650 \mathrm{~nm}$ (the acceptor window) containing both FRET excited mCherry fluorescence and significant non-interacting donor (EGFP) bleed through.
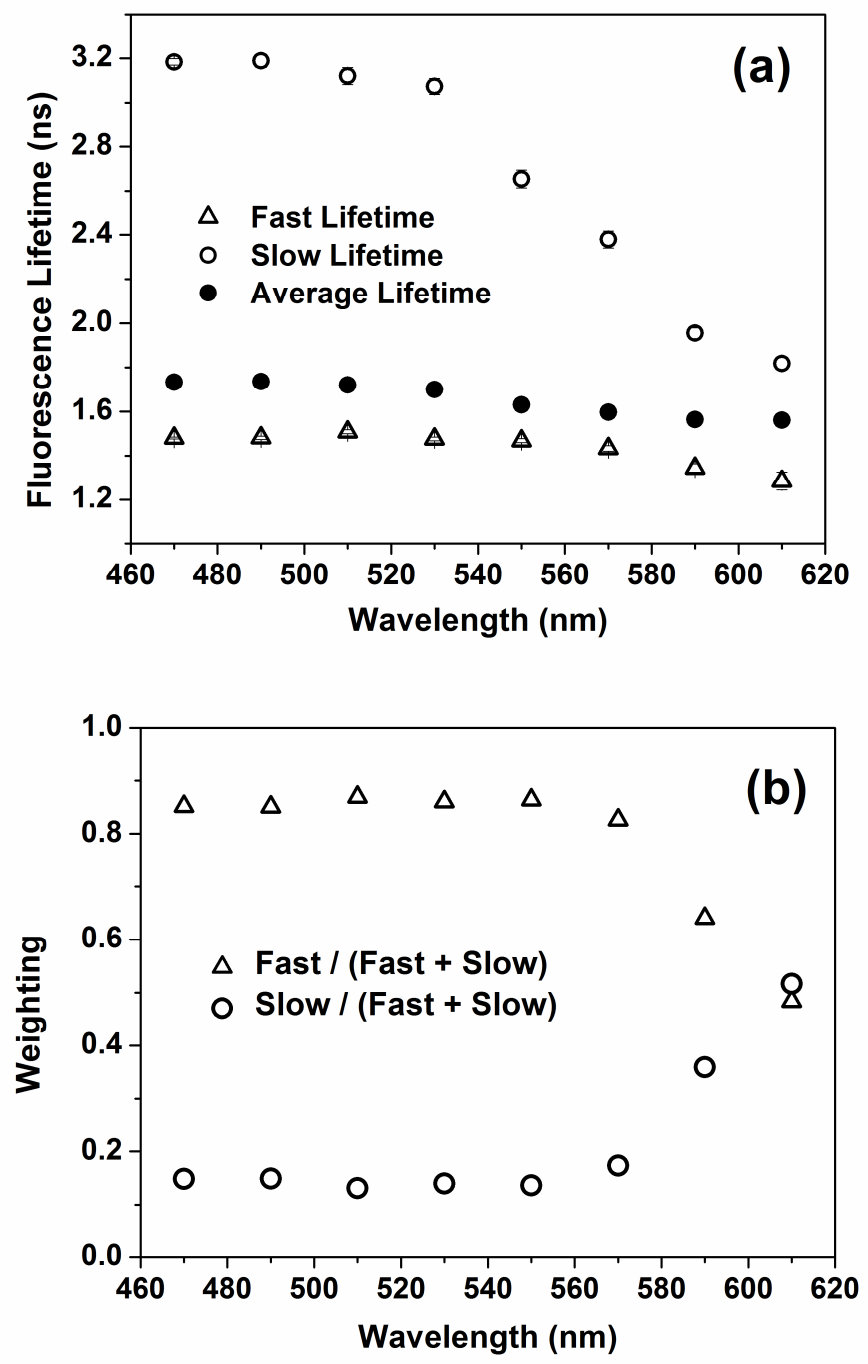

Figure 2: Variation in the acceptor window bi-exponential fluorescence decay characteristics of singlephoton excited mCherry with excitation wavelengths spanning the FRET excitation window of Fig. 1. Reprinted with permission from "Restricted State Selection in Fluorescent Protein Förster Resonance Energy Transfer", Thomas A. Masters, Richard J. Marsh, Daven A. Armoogum, Nick Nicolaou, Banafshé Larijani, and Angus J. Bain, Journal of the American Chemical Society 2013 135 (21), 78837890 DOI: 10.1021/ja312230b, Copyright 2013 American Chemical Society. 
Given the intrinsic heterogeneity in the EGFP and mCherry populations (see Table 1 and Figure 2), a formal description of the FRET dynamics arising from the partial dimerization of PDK1 should in principle involve four pathways. From measurements of the EGFP fluorescence decay we observed that FRET appeared to be restricted to the faster decay component of EGFP ${ }^{9}$ leaving the possibility of FRET from this state accessing the two mCherry populations. If FRET between the "active" state of EGFP and the two mCherry sub-populations was unrestricted, we would expect to observe an acceptor window fluorescence signal that contains both the sensitized acceptor emission arising from FRET together with the bleed through of non-interacting (and non-participating) EGFP emission. The relative sensitivities of the acceptor window to these two signals, the B parameter ${ }^{9}$ (see Equation 1), was calculated to be $19.95^{9}$ so with a $5 \%$ fraction of interacting donors, the relative contribution of sensitized acceptor fluorescence and donor bleed through in the acceptor window are approximately equal.

With approximately equal donor and acceptor contributions to the acceptor window signal the fluorescence, assuming one active donor FRET population and negligible direct acceptor excitation, is potentially complex, having the form:

$$
\begin{aligned}
& I(t) \propto F_{1}^{\mathrm{I}} \exp \left(-\left(k_{\mathrm{F}}^{\mathrm{D}}+k_{\mathrm{FRET} 1}\right) t\right)+F_{2}^{\mathrm{I}} \exp \left(-\left(k_{\mathrm{F}}^{\mathrm{D}}+k_{\mathrm{FRET} 2}\right) t\right) \\
& +B\left[\begin{array}{l}
F_{1}^{\mathrm{I}} X_{1}\left[\exp \left(-k_{\mathrm{F}}^{\mathrm{A} 1} t\right)-\exp \left(-\left(k_{\mathrm{F}}^{\mathrm{D}}+k_{\mathrm{FRET} 1}\right) t\right)\right] \\
+F_{2}^{\mathrm{I}} X_{2}\left[\exp \left(-k_{\mathrm{F}}^{\mathrm{A}} t\right)-\exp \left(-\left(k_{\mathrm{F}}^{\mathrm{D}}+k_{\mathrm{FRET} 2}\right) t\right)\right]
\end{array}\right] \\
& +\left(1-F_{1}^{\mathrm{I}}-F_{2}^{\mathrm{I}}\right) \exp \left(-k_{\mathrm{F}}^{\mathrm{D}} t\right)+C \exp \left(-k_{\mathrm{F}}^{\mathrm{DNI}} t\right)
\end{aligned}
$$

where $\boldsymbol{F}_{1}{ }^{\mathbf{I}}$ and $\boldsymbol{F}_{2}{ }^{\mathrm{I}}$ are the fractions of the active donors that are interacting via FRET with acceptor states 1 and $2, \boldsymbol{k}_{\mathrm{F}}{ }^{\boldsymbol{D}}$ is the fluorescence decay rate of the non-interacting but FRET active donor, $\boldsymbol{k}_{\mathrm{F}}{ }^{\mathbf{A I}}$ and $\boldsymbol{k}_{\mathrm{F}}{ }^{\mathrm{A} 2}$ are the fluorescence decay rates of the two excited states of the acceptor, $\boldsymbol{k}_{\mathrm{FRET}}$ and $\boldsymbol{k}_{\text {FRET2 }}$ are the rates at which FRET occurs from an active donor to each of the acceptor states, $\boldsymbol{k}_{\mathbf{F}}{ }^{\text {DNI }}$ is the fluorescence decay rate of the FRET inactive donor population and $\boldsymbol{C}$ is the ratio of inactive to active donors. The corresponding fluorescence lifetimes are given by the inverse of the total decay rates of each species. The $\boldsymbol{X}$ parameters are given by,

$$
X_{1}=\frac{k_{\mathrm{FRET} 1}}{\boldsymbol{k}_{\mathrm{F}}^{\mathrm{D}}+\boldsymbol{k}_{\mathrm{FRET} 1}-\boldsymbol{k}_{\mathrm{F}}^{\mathrm{A} 1}} \text { and } X_{2}=\frac{\boldsymbol{k}_{\mathrm{FRET} 2}}{\boldsymbol{k}_{\mathrm{F}}^{\mathrm{D}}+\boldsymbol{k}_{\mathrm{FRET} 2}-\boldsymbol{k}_{\mathrm{F}}^{\mathrm{A} 2}}
$$

With a $\boldsymbol{B}$ value in the range of $20^{9}$ and a small interacting fraction, the dominant terms in the acceptor window intensity are

$$
\begin{aligned}
& I(t) \propto B {\left[\begin{array}{l}
F_{1}^{\mathrm{I}} X_{1}\left[\exp \left(-k_{\mathrm{F}}^{\mathrm{A} 1} t\right)-\exp \left(-\left(k_{\mathrm{F}}^{\mathrm{D}}+k_{\mathrm{FRET} 1}\right) t\right)\right] \\
+F_{2}^{\mathrm{I}} X_{2}\left[\exp \left(-k_{\mathrm{F}}^{\mathrm{A}} t\right)-\exp \left(-\left(k_{\mathrm{F}}^{\mathrm{D}}+k_{\mathrm{FRET} 2}\right) t\right)\right]
\end{array}\right.} \\
&+\left(1-F_{1}^{\mathrm{I}}-F_{2}^{\mathrm{I}}\right) \exp \left(-k_{\mathrm{F}}^{\mathrm{D}} t\right)+C \exp \left(-k_{\mathrm{F}}^{\mathrm{DNI}} t\right)
\end{aligned}
$$

Qualitatively the acceptor window fluorescence is characterized by a growth (the negative amplitude exponential terms in Equation 3) and subsequent decay. In the case of positive $\mathrm{X}$ values, this originates from the non-interacting donor bleed through and the decay of FRET excited mCherry. Extracting six lifetime components from the acceptor window fluorescence is not a realistic proposition. Even with high signal to noise ratio data, a biexponential fit to the acceptor window data (yielding an effective rise time and an overall decay time) is the most accurate analysis that be achieved ${ }^{9,26}$. In order to extract more information on the nature of the FRET interaction, additional experimental approaches are necessary. 


\subsection{Acceptor Window Fluorescence Anisotropy Measurements}

Fluorescence anisotropy measurements are a most useful counterpart to intensity data. In a heterogeneous system with $\boldsymbol{i}$ components, the net fluorescence anisotropy ${ }^{27}$ is given by

$$
R(t, \lambda)=\sum_{i} W_{i}(t, \lambda) R_{i}(t)
$$

where $\boldsymbol{W}_{\boldsymbol{i}}(\boldsymbol{t}, \lambda)$ is the time-dependent weighting factor of component $\boldsymbol{i}$ at a particular emission wavelength $\lambda$,

$$
W_{i}(t, \lambda)=\alpha_{i}(\lambda) F_{i}(t) / \sum_{j} \alpha_{j}(\lambda) F_{i}(t)
$$

Here $\alpha_{i}(\lambda)$ is the initial detection amplitude of species $i$ at emission wavelength $\lambda$ and is dependent on the initial population size and the spectral detection efficiency of the apparatus and $\boldsymbol{F}_{i}(\boldsymbol{t})$ represents the intrinsic population dynamics of species $\boldsymbol{i}$. With direct optical excitation the $\boldsymbol{F}_{i}(\boldsymbol{t})$ are single exponential decay functions. However for FRET transfer, the $\boldsymbol{F}_{\boldsymbol{i}}(\boldsymbol{t})$ have the bi-exponential functional form as in Equation 3. In the donor window, the composite fluorescence anisotropy arises from that of the interacting and non-interacting donor populations, whilst the acceptor window signal is dominated by the non-interacting donor bleed through and the sensitized acceptor fluorescence arising from FRET. In the PDKI dimerization system, the intrinsic anisotropy decays arising from rotational diffusion are slow. The donor bleed through is characterized by a high initial anisotropy, $R(0) \approx 4 / 7$, and a slow rotational diffusion time $\left(\boldsymbol{t}_{\mathbf{R}}{ }^{\mathbf{D N I}}\right)$ of ca $30 \mathrm{~ns}^{9}$, and for the fluorescently tagged homodimer the rotational diffusion time $\left(\boldsymbol{t}_{\mathbf{R}}{ }^{\mathbf{A}}\right)$ should be significantly higher. Changes in the fluorescence anisotropy in the $12 \mathrm{~ns}$ time window of Figure 3 therefore arise from changes in the relative weighting of the intrinsic non-interacting donor anisotropies,

$$
R_{D}^{N I}(t) \cong \frac{4}{7} \exp \left(-t / t_{R}^{D N I}\right) \quad \text { and } \quad R_{A 1,2}(t) \cong \frac{4}{7} P_{2}\left(\cos \gamma_{1,2}\right) \exp \left(-t / t_{R 1,2}^{A}\right)
$$

Here $\gamma_{1,2}$ is the molecular frame rotation angle of the emission transition dipole moment between the donor and the two possible acceptor states. The rapid (sub-ns) depolarization of the acceptor window anisotropy therefore corresponds to the effective rotation of the emitting transition dipole moment in the molecular frame as a result of FRET ${ }^{9}$ with $\gamma_{1,2}>54.7^{\circ}$ such that $\boldsymbol{P}_{\mathbf{2}}\left(\cos \gamma_{1,2}\right)$ is negative. If FRET were unrestricted, we would expect the acceptor population to be characterized by a bi-exponential decay with lifetimes of $1.47 \mathrm{~ns}$ (ca 84\%) and $2.77 \mathrm{~ns}$ (ca 16\%) respectively. The excited mCherry population resulting from FRET would therefore be expected to decay with an approximate lifetime of $1.67 \mathrm{~ns}$ (see above). The average fluorescence lifetime of EGFP in the donor window is $2.75 \mathrm{~ns}{ }^{9}$. The relative weighting of mCherry fluorescence to EGFP bleed through should first increase from zero on the timescale of FRET transfer leading to a drop in the composite anisotropy (as observed in Figure 3). However, if laboratory frame photoselection and FRET were wholly equivalent (i.e. the population of both mCherry emitting states), the overall mCherry weighting will decrease leading to a rise in the anisotropy within a few ns as the non-interacting donor bleed through predominates. This is clearly not the case, and to maintain the low fluorescence anisotropy that is observed, the sensitized mCherry fluorescence must have a similar lifetime to that of the non-interacting EGFP bleed through $(2.75 \mathrm{~ns})$. FRET must therefore take place exclusively to the minority $2.77 \mathrm{~ns}$ decay (15\%) component of the mCherry population. 


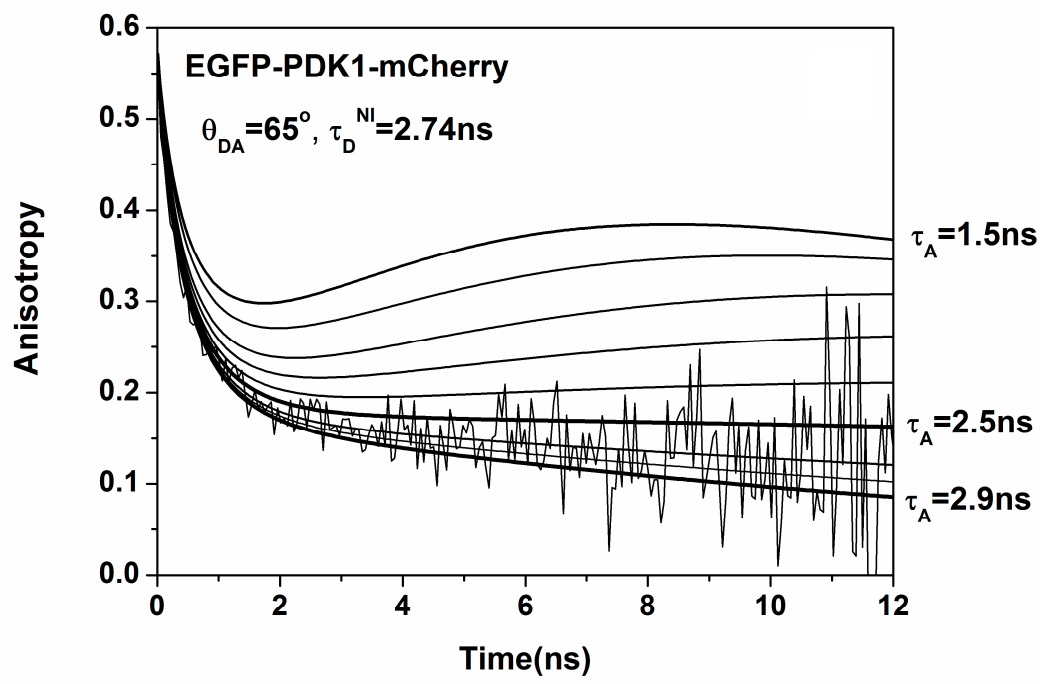

Figure 3: The EGFP-PDK1-mCherry acceptor window anisotropy decay following two-photon donor excitation at $880 \mathrm{~nm}$. The observed anisotropy is the population weighted contribution of the sensitized acceptor anisotropy and the fluorescence anisotropy from the bleed through of non-interacting donor fluorescence. The model anisotropy decays (solid lines) show the dependence of the composite anisotropy on the lifetime of FRET excited mCherry for an interchromophore (depolarization) angle of $65^{\circ}$. Reprinted with permission from "Restricted State Selection in Fluorescent Protein Förster Resonance Energy Transfer", Thomas A. Masters, Richard J. Marsh, Daven A. Armoogum, Nick Nicolaou, Banafshé Larijani, and Angus J. Bain, Journal of the American Chemical Society 2013135 (21), 7883-7890 DOI: 10.1021/ja312230b, Copyright 2013 American Chemical Society.

\section{AN ALTERNATIVE APPROACH TO SENSITIZED FLUORESCENCE INTENSITY MEASUREMENTS}

FRET is most commonly detected and analyzed through the measurement of the donor fluorescence intensity decay, with measurement of the reduction in the donor lifetime due to FRET yielding the transfer rate if the intrinsic fluorescence decay rate is known ${ }^{28,29}$. Additionally, if the $\kappa^{2}$ orientation parameter and the Förster radius are known, the donor-acceptor distance can be determined ${ }^{30}$.

For a homogeneous population of interacting donor and acceptor molecules, Equations 1 and 2 simplify to,

$$
\begin{gathered}
I(t) \propto B F_{I} X\left[\exp \left(-k_{\mathrm{F}}^{\mathrm{A}} t\right)-\exp \left(-\left(k_{\mathrm{F}}^{\mathrm{D}}+k_{\mathrm{FRET}}\right) t\right)\right]+\left(1-F_{1}\right) \exp \left(-k_{\mathrm{F}}^{\mathrm{D}} t\right)+F_{I} \exp \left(-\left(k_{\mathrm{F}}^{\mathrm{D}}+k_{\mathrm{FRET}}\right) t\right) \\
X=k_{\mathrm{FRET}} /\left(k_{\mathrm{F}}^{\mathrm{D}}+k_{\mathrm{FRET}}-k_{\mathrm{F}}^{\mathrm{A}}\right)
\end{gathered}
$$

Thus, when $\boldsymbol{k}_{\text {FRET }}$ is greater than the difference between the donor fluorescence rate and that of the acceptor, the $\boldsymbol{X}$ parameter is positive and the sensitized acceptor fluorescence will exhibit an exponential rise whose time constant is $\left(\boldsymbol{k}_{\mathrm{F}}^{\mathbf{D}}+\boldsymbol{k}_{\mathrm{FRET}}\right)^{-1}$. Measurement of this rise lifetime (given $\left.\boldsymbol{k}_{\mathrm{F}} \mathbf{D}\right)$ should therefore permit the determination of $\boldsymbol{k}_{\text {FRET }}$. As such, this approach has been has been widely applied ${ }^{23,31-35}$. In the previous section we showed how the interplay between the non-interacting donor bleed through and that of the sensitized acceptor emission can yield a composite fluorescence anisotropy signal that is very sensitive to lifetime differences between the two species if both 
populations are characterized by slowly relaxing intrinsic orientational correlation functions but with fluorescence anisotropies of opposite sign (arising from FRET dipole rotation angles of $\gamma>54.7^{\circ}$ ). However, this is not the case for intensity measurements, as the combination of sensitized acceptor fluorescence and non-interacting donor bleed through is always additive as opposed to subtractive.

FRET restriction between EGFP and mCherry in the PDK1 dimerization system leads to sensitized acceptor window fluorescence given by Equation 7. Least squares fitting analysis of the acceptor window fluorescence decay, including iterative reconvolution with the instrument response function, yielded a bi-exponential signal with one positive and one negative amplitude component,

$$
\begin{aligned}
I(t) \propto A_{\text {decay }} \exp \left(-\frac{t}{\tau_{\text {decay }}}\right)-A_{\text {rise }} \exp \left(-\frac{t}{\tau_{\text {rise }}}\right) \\
=A_{\text {decay }}\left(\exp \left(-\frac{t}{\tau_{\text {decay }}}\right)-\left|\alpha_{\text {rise }}\right| \exp \left(-\frac{t}{\tau_{\text {rise }}}\right)\right)
\end{aligned}
$$

However, the rise and decay lifetimes extracted did not unequivocally indicate the FRET restrictions that were found to operate, necessitating the use of the acceptor window anisotropy to provide explicit confirmation of the underlying population dynamics. Acceptor anisotropy measurements give the clearest picture of population dynamics when the interacting species do not undergo any significant orientational depolarization. However, these dynamics are significantly more complex for the case of a rapidly reorienting donor interacting with a slowly reorienting acceptor ${ }^{36,37}$. In these situations a reappraisal of the information that can be extracted from acceptor window intensity measurements becomes necessary.

Bi-exponential models as in Equation 9 appear to provide good agreement with acceptor window fluorescence intensity data, based on satisfactory $\boldsymbol{\chi}_{\mathbf{R}}{ }^{2}$ values. However, as shown in Equation 1, more decay rates than the two provided by the fit will contribute to the shape of the decay. The relationship of the parameters extracted from bi-exponential fitting to the underlying heterogeneous population dynamics and non-interacting donor bleed through must therefore be assessed. We therefore performed numerical simulations of FRET from a homogeneous donor (single lifetime of $4 \mathrm{~ns}$ ) and heterogeneous acceptor population with lifetimes of $1.315 \mathrm{~ns}$ and $1.897 \mathrm{~ns}$, mirroring those observed in a recent study in our laboratory ${ }^{26}$. Under these circumstances, the parameter $\mathbf{C}$ in equation 1 becomes zero. As $\boldsymbol{X}$ values can become large and negative in the vicinity of $\boldsymbol{k}_{\mathrm{FRET}}+\boldsymbol{k}_{\mathrm{F}}{ }^{\mathrm{D}} \approx \boldsymbol{k}_{\mathrm{F}}{ }^{\mathrm{A}}\left(\boldsymbol{\tau}_{\mathrm{FRET}} \approx \boldsymbol{\tau}_{\mathrm{A}}\right)$, simulations were performed using six values of the interacting donor lifetime close to and in-between the acceptor lifetimes. To investigate the impact of the amount of FRET occurring on the parameters output, interacting fractions of $0.25,0.50,0.75$ and 1.00 were simulated, assuming equal abundance of the two acceptor species and equal FRET rates to the two states. The amount of donor bleed through into the acceptor window was controlled by varying $\boldsymbol{B}$ between 0.5 and 100 .

Fluorescence decay datasets were simulated using MATLAB (The Mathworks) by convoluting the fluorescence decay function with the measured IRF of our TCSPC system ${ }^{9}$. Poisson noise of a magnitude expected for measurements with a peak value of 5000 photons was then added, to reflect the signal levels that we obtain in our measurements. The decay was then fit to a biexponential function using iterative reconvolution with the IRF in the Fluofit MATLAB package ${ }^{38}$. A good fit was judged to be that which minimised the $\boldsymbol{\chi}_{\mathbf{R}}{ }^{2}$ statistic below 2. 10 decays were simulated at each $\boldsymbol{B}$ value for each set of FRET parameters, giving a total of 24,000 fluorescence decays. This process is schematically represented in Figure 4. 


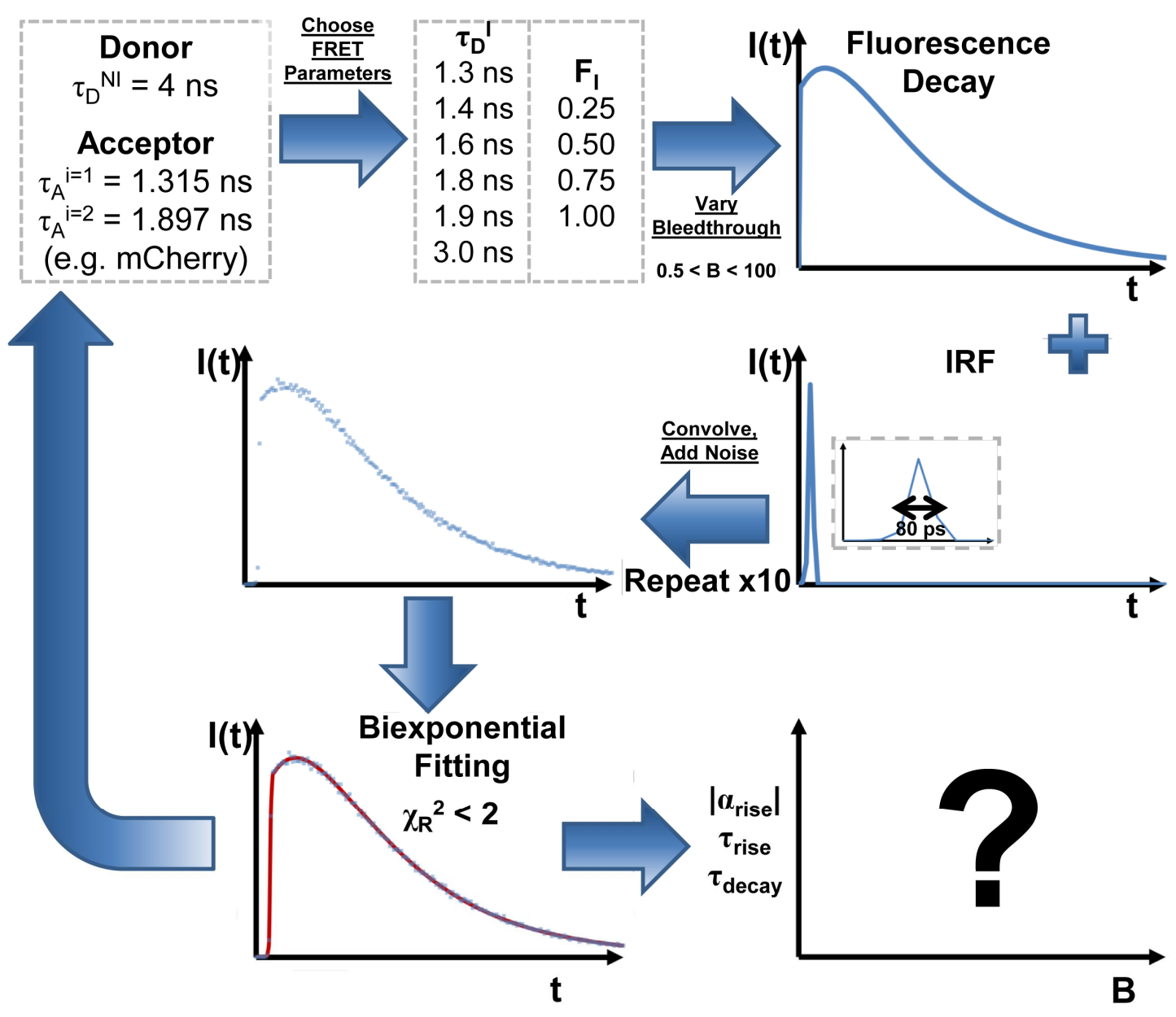

Figure 4: Schematic approach to acceptor window fluorescence fit modelling. After choice of FRET parameters, 10 fluorescence decays were simulated and biexponentially fit for $200 \boldsymbol{B}$ values between 0.5 and 100 , allowing the extracted parameters $\left|\alpha_{\text {rise }}\right|, \tau_{\text {rise }}$ and $\tau_{\text {decay }}$ to be compared with those expected from theory. 
The results of these simulations are shown in Figure 5. We observed that the ratio of the magnitude of the negative decay amplitude to the positive amplitude, $\mid \boldsymbol{\alpha}_{\text {rise }}$, increases towards the limiting value of 1 with increasing $\boldsymbol{B}$. From Equation 1 this would be expected as for large $\boldsymbol{B}\left(\right.$ or $\left.\mathbf{F}_{\mathbf{I}}\right)$ the positive amplitude bleed through in the acceptor window can be neglected, and each positive amplitude FRET decay component will be present with a negative component of equal magnitude. We therefore investigated whether the variation in $\left|\boldsymbol{\alpha}_{\text {rise }}\right|$ with $\boldsymbol{B}$ could be described by the ratio of the negative amplitudes to the positive amplitudes present in Equation 1, given by

$$
\left|\alpha_{\text {rise }}\right|=\frac{B\left(F_{1}^{\mathrm{I}} X_{1}+F_{2}^{\mathrm{I}} X_{2}\right)}{B\left(F_{1}^{\mathrm{I}} X_{1}+F_{2}^{\mathrm{I}} X_{2}\right)+1}
$$

Good agreement between Equation 10 and the simulated data would imply that the rates of FRET to the two acceptor states could be deduced from the ratio of amplitudes alone, allowing state restriction to be investigated using this parameter. Figures 5(a) \& 5(b) show the worst and best agreements of this function and the output of the computational model, where it can be seen that Equation 10 and the amplitude ratio extracted by fitting did not agree at the smaller values of $\boldsymbol{B}$ (5 to 20 ) typically obtainable in experimental studies. Averaged across all $\boldsymbol{B}$ values, the simulated data disagreed with that expected from Equation 10 by between $0.6 \%$ (Figure 5(b)) and $12.7 \%$ (Figure 5(a)). The best agreement occurred with all donors interacting with a lifetime of $1.4 \mathrm{~ns}$ with the worst occurring with an interacting donor lifetime of $1.9 \mathrm{~ns}$ and a low interacting fraction of 0.25 . The variation in agreement between model predictions and the best-fit amplitudes with interacting fraction and donor lifetime is shown in Figure 5(a). Here it can be seen that the agreement between the fit amplitudes and Equation 10 increases with increasing interacting fraction, with the worst agreements occurring with interacting donor lifetimes close to the lifetimes of the acceptor states. These correspond to the situations at which the transfer amplitude $\boldsymbol{X}$ can become very large in either the positive or negative direction.

The relationship between amplitude-weighted averages of the negative amplitude and positive amplitude lifetimes present in Equation 1 and $\boldsymbol{\tau}_{\text {rise }}$ and $\boldsymbol{\tau}_{\text {decay }}$ obtained from fits to the simulated data was also investigated. The best and worst agreements for these parameters across all $\boldsymbol{B}$ values are shown in Figures 5(c)-(d) and 5(e)-(f). Again, averaged across all $\boldsymbol{B}$ values (Figures 6(b) \& 6(c)), disagreements were largest at large $\boldsymbol{X}$ values when the interacting donor lifetime was close to that of the acceptor lifetimes $\left(\boldsymbol{k}_{\mathrm{FRET}}+\boldsymbol{k}_{\mathrm{F}}^{\mathbf{D}} \approx \boldsymbol{k}_{\mathrm{F}}{ }^{\mathbf{A}}\right)$. For all values of the FRET parameters, the rise lifetime was shorter than that expected from an amplitude-weighted average of the negative amplitude components. Likewise, the best-fit decay lifetime was larger than an amplitude-weighted average of the positive amplitude components in the acceptor window decay model.

These results demonstrated that none of the fit parameters could individually be used to extract the unknown parameters from Equation 1 from acceptor window fluorescence intensity decay data. The disagreements between simple amplitude-weighted models of each parameter and the parameters output by the fits were largest at high donor bleed through (low $\boldsymbol{B}$ ). This suggested that the presence of increased donor bleed through (no negative amplitude component) was skewing the bi-exponential fit parameters towards shorter rise times and correspondingly longer decays times, with their relative amplitudes altered in order to maintain a good fit to the data. Despite the lack of correlation between individual best-fit parameters and the parameters describing the FRET interaction in the theoretical model, good fits across the decay curves were nevertheless maintained. As the individually skewed parameters still gave a good description of the shape of the acceptor window fluorescence decay, we investigated the agreement between a composite function of the three decay parameters and that expected from the theoretical model of FRET. 

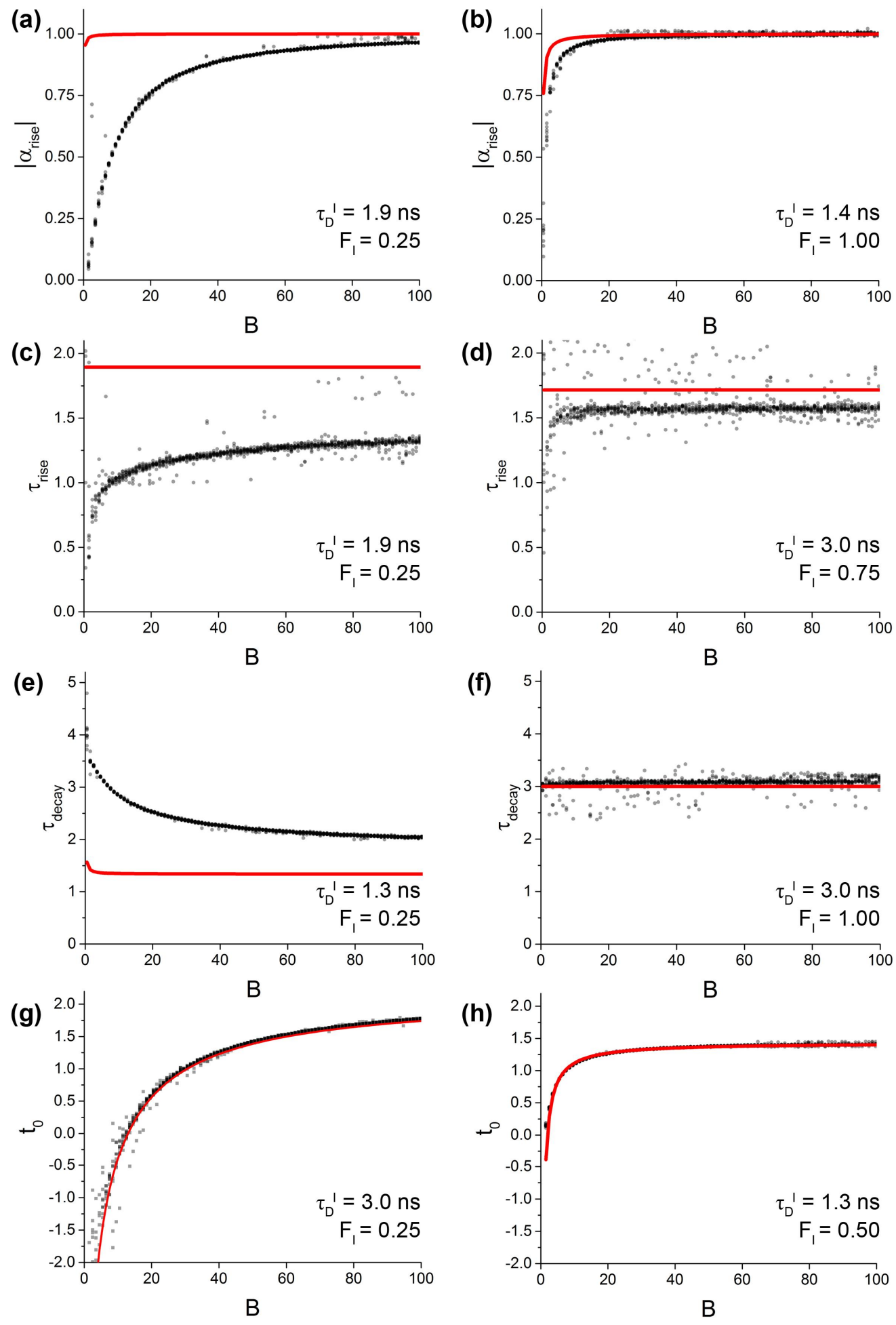

Figure 5: Worst (left) and best (right) agreements between each parameter extracted from least-squares fitting (black points) and their corresponding amplitude-weighted approximation (red lines). 

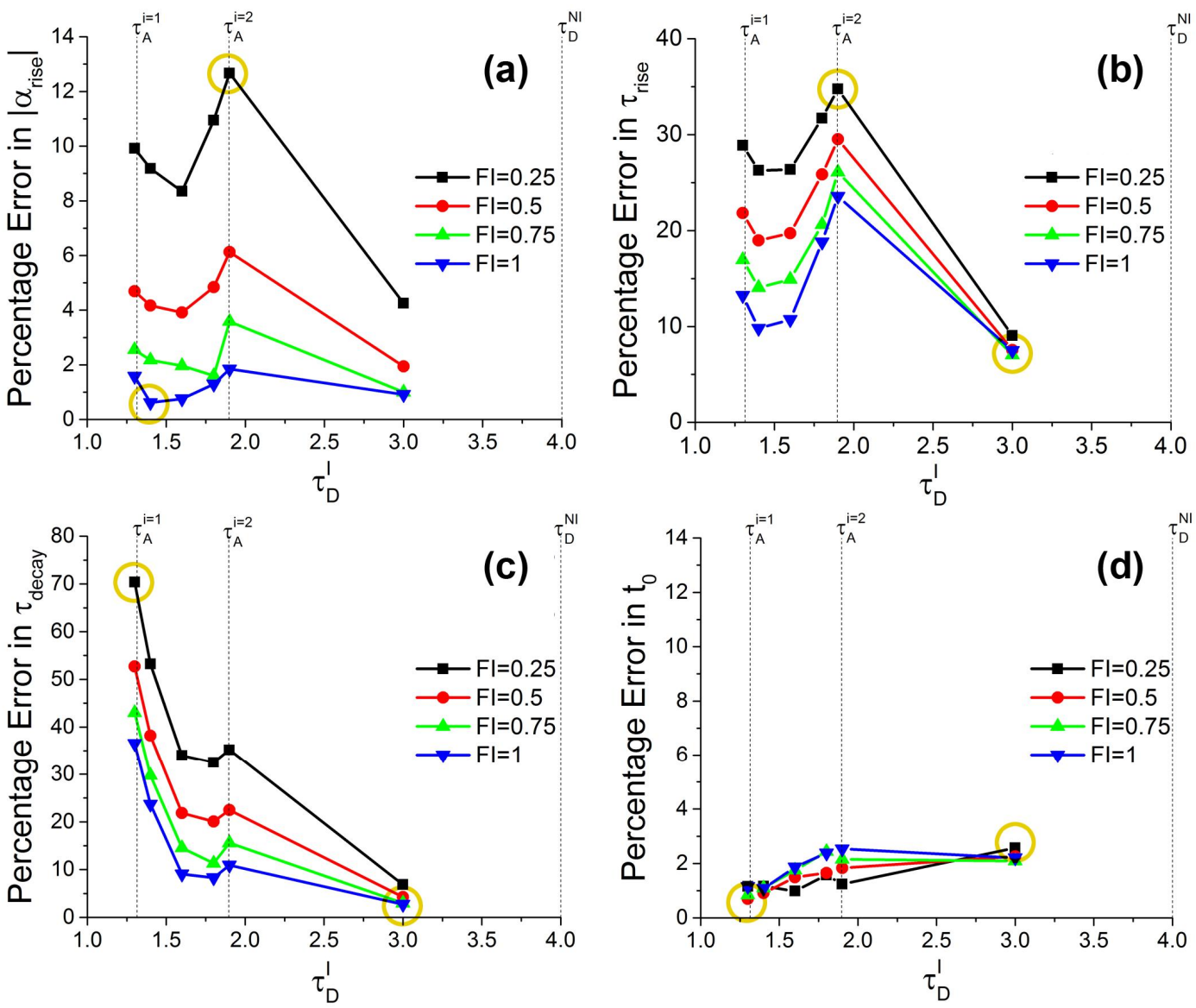

Figure 6: Mean disagreement across all $\boldsymbol{B}$ values between parameters extracted from least-squares fitting and their corresponding amplitude-weighted approximations at each simulated value of the interacting donor lifetime. For each parameter, the values of $\boldsymbol{\tau}_{\mathbf{D}}{ }^{\mathbf{I}}$ and $\boldsymbol{F}_{\mathbf{I}}$ giving the best and worst agreements, shown in Figure 5, are circled.

As shown in Figure 4, the shape of the acceptor window fluorescence decay is characterized by an initial rise and subsequent decay. The turnover between these regimes takes place at time $\boldsymbol{t}_{\mathbf{0}}$ when the rise (negative amplitude) and decay (positive amplitude) components in Equation 1 are equal in magnitude. Increased donor bleed through will therefore cause $\boldsymbol{t}_{\mathbf{0}}$ to shift to earlier times. The point at which the best-fit curve turns over is a function of all three fit parameters, achieved by differentiation of the biexponential fitting function, giving,

$$
t_{0}=\frac{\tau_{\text {rise }} \tau_{\text {decay }}}{\tau_{\text {decay }}-\tau_{\text {rise }}} \ln \left(\frac{\left|\alpha_{\text {rise }}\right| \tau_{\text {decay }}}{\tau_{\text {rise }}}\right)
$$


For the theoretical model, the turnover point $\boldsymbol{t}_{0}$ would be given by the numerical solution of,

$$
\begin{aligned}
& \left(F_{1}^{I}+F_{2}^{I}-1\right) k_{F}^{D} \exp \left(-k_{F}^{D} t_{0}\right) \\
& -F_{1}^{I}\left(k_{F}^{D}+k_{\mathrm{FRET} 1}\right) \exp \left(-\left(k_{F}^{D}+k_{\mathrm{FRET} 1}\right) t_{0}\right) \\
& -F_{2}^{I}\left(k_{F}^{D}+k_{\mathrm{FRET} 2}\right) \exp \left(-\left(k_{F}^{D}+k_{\mathrm{FRET} 2}\right) t_{0}\right) \\
& =B\left\{\begin{array}{l}
F_{1}^{I} X_{1}\left[k_{F}^{A 1} \exp \left(-k_{F}^{A 1} t_{0}\right)-\left(k_{F}^{D}+k_{\mathrm{FRET} 1}\right) \exp \left(-\left(k_{F}^{D}+k_{\mathrm{FRET} 1}\right) t_{0}\right)\right] \\
+F_{2}^{I} X_{2}\left[k_{F}^{A 2} \exp \left(-k_{F}^{A 2} t_{0}\right)-\left(k_{F}^{D}+k_{\mathrm{FRET} 2}\right) \exp \left(-\left(k_{F}^{D}+k_{\mathrm{FRET} 2}\right) t_{0}\right)\right]
\end{array}\right\}
\end{aligned}
$$

We therefore investigated whether the composite fit parameter in Equation 11 gave a better agreement with the theory in Equation 12 than any of the individually skewed parameters. As shown in Figures $5(\mathrm{~g}), 5(\mathrm{~h})$ and $6(\mathrm{e})$, the turnover point of the least-squares fit and the theoretical FRET model were in excellent agreement for all FRET interaction parameter values, suggesting that in instances where the acceptor window anisotropy cannot be easily used to determine the FRET population dynamics, determination of the turning point of the acceptor window intensity decay provides a useful alternative.

\section{CONCLUSIONS}

It is clear from both experimental work and theoretical modeling that the unavoidable complexity of intrinsic and environmental heterogeneity in fluorescent protein FRET cannot be adequately addressed solely through conventional fluorescence intensity decay measurements. In systems where donor and acceptor rotational motion is slow compared to the timescale of FRET, the relative weighting of the non-interacting donor bleed through and the sensitized acceptor emission gives rise to acceptor window anisotropy dynamics that are sensitive to the lifetimes of the acceptor states accessed. This information, together with conventional donor and acceptor window intensity decay data, has been successfully applied in unraveling the FRET dynamics of the PDK1 dimerization system. The use of anisotropy data in more orientationally mobile donor-acceptor systems is less straightforward and an alternative approach to extracting real FRET rates through the measurement of the turn over time in the acceptor window emission signal has been demonstrated by theoretical modeling. Quantitative and reliable FRET measurements in heterogeneous systems should therefore be possible through a combination of these approaches.

\section{ACKNOWLEDGEMENTS}

We would like to thank the BBSRC for support of this work.

\section{REFERENCES}

[1] B. Albinsson and J. K. Hannestad, "Multistep FRET and nanotechnology", in FRET- Förster Resonance Energy Transfer: From Theory to Applications (eds I. Medintz and N. Hildebrandt), WileyVCH Verlag GmbH \& Co. KGaA, Weinheim, Germany (2013)

[2] B. Schuler, W. A. Eaton, "Protein folding studied by single-molecule FRET", Curr. Op. in Struct. Biol., 18, 16-26 (2008)

[3] C. M. Dalton, G. Szabadkai and J. Carroll, "Measurement of ATP in single oocytes: impact of maturation and cumulus cells on levels and consumption", J. Cell. Physiol., 229 353-361 (2014) 
[4] L. Stryer and R. P. Haugland, "Energy transfer: a spectroscopic ruler", Proc. Nat. Acad Sci USA, $58,719-726(1967)$

[5] A. A. Deniz, M. Dahan, J. R. Grunwell, T. Ha, A. E. Faulhaber, D. S. Chemla, S Weiss and P.G. Schultz, "Single-pair fluorescence resonance energy transfer on freely diffusing molecules: Observation of Förster distance dependence and subpopulations", Proc. Nat.. Acad. Sci. USA, 96, 3670-3675 (1999)

[6] J. Zhang R. E. Campbell, A. Y. Ting, and R. Y. Tsien, "Creating new fluorescent probes for cell biology”, Nature Revs. Mol. Cell Biol. 3, 906-918 (2002)

[7] D. W. Piston, G-J. Kremers, "Fluorescent protein FRET: the good, the bad and the ugly", Trends in Biochem. Sci. 32, 407-414 (2007)

[8] M. Zimmer "GFP: from jellyfish to the Nobel prize and beyond" Chem. Soc. Rev. 38 2823-2832 (2009)

[9] T. A. Masters, R. J. Marsh, D. A. Armoogum, N. Nicolaou, B. Larijani and A. J. Bain, "Restricted state selection in fluorescent protein Förster resonance energy transfer", J. Am. Chem. Soc. $1357833-$ 7890 (2013)

[10] T. A. Masters, V. Calleja, D. A. Armoogum, R. J Marsh, C. J. Applebee, M Laguerre, A. J. Bain and B. Larijani, "Regulation of 3-phosphoinositide-dependent protein kinase 1 activity by homodimerization in live cells", Sci. Signal. 3143 ra78 (2010)

[11] A. J. W. G. Visser, S. P. Laptenok N.V. Visser, A. van Hoek, J.-C. Brochon and J.W. Borst, "Time-resolved FRET fluorescence spectroscopy of visible fluorescent protein pairs", Eur. Biophys. $J$. 39 241-253 (2010)

[12] S.T. Hess E. D. Sheets, A. Wagenknecht-Wiesner and A. A. Heikal, "Quantitative analysis of the fluorescence properties of intrinsically fluorescent proteins in living cells", Biophysical J., 85 25662580 (2003)

[13] S. Lee, S. Y. Kim, K. Park, B. H. Chung, S. W. Kim "Fluorescence lifetime study of ECFP/EYFP labeled MBP in Förster resonance energy transfer", J. Lumin.131 275-279 (2011)

[14] P. Sarkar, S. V. Koushik, S. S. Vogel, "Photophysical properties of Cerulean and Venus fluorescent proteins", J. Biomed. Opt. 14, 034047 1-9 (2009)

[15] J. W. Borst, M. A. Hink, A. van Hoek and A. J. W. G. Visser, "Effects of refractive index and viscosity on fluorescence and anisotropy decays of enhanced cyan and yellow fluorescent proteins", $J$. Fluorescence 15, 153-160 (2005)

[16] A. A. Heikal, S. T. Hess W. W. Webb, "Multiphoton molecular spectroscopy and excited-state dynamics of enhanced green fluorescent protein (EGFP): acid-base specificity", Chem. Phys 274 37-55 (2001)

[17] M. Tramier, I. Gautier, T. Piolot, S. Ravalet, K. Kemnitz, J. Coppey, C. Durieux, V. Mignotte, M. Coppey-Moisan, "Picosecond hetero-FRET microscopy to probe protein-protein interactions in live cells”, Biophys. J. 83 3570-3577 (2002).

[18] R. R. Duncan, A. Bergmann, M. A. Cousin, D. K. Apps, M. J. Shipston, "Multidimensional timecorrelated single photon counting (TCSPC) fluorescence lifetime imaging microscopy (FLIM) to detect FRET in cells", J. Microsc. 215, 1-12 (2004) 
[19] M. Millington G. J. Grindlay, K. Altenbach R. K. Neely, W. Kolch, M. Bencina, N. D. Read, A. C. Jones D. T. F. Dryden and S. W. Magennis, "High-precision FLIM-FRET in fixed and living cells reveals heterogeneity in a simple CFP-YFP fusion protein", Biophys. Chem. 127, 155-164 (2007)

[20] S. S. Vogel T. A. Nguyen, B. W. van der Meer and P. S. Blank, "The impact of heterogeneity and dark acceptor states on FRET: implications for using fluorescent protein donors and acceptors", PLoS ONE 7: e49593 (2012)

[21] J. Arpino, P. Rizkallah and D. D. Jones, "Crystal structure of enhanced green fluorescent protein to $1.35 \AA$ resolution reveals alternative conformations for Glu222", PLoS ONE 7: e47132 (2012)

[22] N. Akrap, T. Seidel, B. G. Barisas, "Förster distances for fluorescence resonant energy transfer between mCherry and other visible fluorescent proteins", Anal. Biochem, 402, 105-106 (2010)

[23] S. P. Laptenok, J. W. Borst, K. M. Mullen, I. H. M. van Stokkum, A. J. W. G. Visser., H. van Amerongen, "Global analysis of Förster resonance energy transfer in live cells measured by fluorescence lifetime imaging microscopy exploiting the rise time of acceptor fluorescence", Phys. Chem. Chem. Phys., 12, 7593-7602 (2010)

[24] X. Shu, N. C. Shaner, C. A. Yarbrough, R. Y. Tsein and S. Remington, "Novel chromophores and buried charges control color in mfruits", Biochemistry 45, 9639-9647(2006)

[25] D. L. Andrews, "Resonance energy transfer: theoretical foundations and developing applications". In: Tutorials in Complex Photonic Media. SPIE Press, Bellingham, WA, 439-478 (2009)

[26] T. S. Blacker, W. Y. Chen, R. J. Marsh, C. F. Kaminski and A. J. Bain., "Investigating state selection in fluorescent protein förster resonance energy transfer using time-resolved fluorescence and anisotropy" (to be published)

[27] R. D. Ludescher, L. Peting, S Hudson and B. Hudson, "Time-resolved fluorescence anisotropy for systems with lifetime and dynamic heterogeneity", Biophys. Chem. 28, 59-75 (1987)

[28] W. Becker, A. Bergmann, M.A. Hink, K. König, K. Benndorf, C. Biskup, "Fluorescence lifetime imaging by time-correlated single-photon counting", Microsc. Res. Tech. 63, 58-66 (2004)

[29] B. Valeur, Molecular Fluorescence: Principles and Applications. 2001 Wiley-VCH Weinheim

[30] VanBeek, D. B., Zwier, M. C., Shorb, J. M., \& Krueger, B. P. "Fretting about FRET: correlation between $\kappa$ and R", Biophysical J., 92, 4168-4178 (2007)

[31] S. Lindhoud, A. H. Westphal, C. P. M. van Mierlo, A. J. W. G. Visser and J. W. Borst, "Rise-time of FRET-acceptor fluorescence tracks protein folding", Int. J. Mol. Sci., 15, 23836-23850 (2014)

[32] S. P. Laptenok, J.W. Borst, K. M. Mullen, I. H. van Stokkum, A. J. Visser, H. van Amerongen, "Global analysis of Förster resonance energy transfer in live cells measured by fluorescence lifetime imaging microscopy exploiting the rise time of acceptor fluorescence", Phys. Chem. Chem. Phys., 12, 7593-7602 (2010)

[33] T. Kulinski, A. J. Visser, D. J. O'Kane and J. Lee "Spectroscopic investigations of the single tryptophan residue and of riboflavin and 7-oxolumazine bound to lumazine apoprotein from photobacterium leiognathi", Biochemistry, 26, 540-549 (1987)

[34] B. Jain, K. Das, "Fluorescence resonance energy transfer between DPH and nile red in a lipid bilayer", Chem. Phys. Lett., 433 170-174 (2006) 
[35] M. Suresh, A. K. Mandal, E. Suresh and A. Das, "First demonstration of two-step FRET in a synthetic supramolecular assembly”, Chem. Sci., 4, 2380-2386 (2013)

[36] F Tanaka and N Mataga, "Dynamic depolarization of interacting fluorophores. Effect of internal rotation and energy transfer”, Biophys J., 39 129-140 (1982)

[37] F. Tanaka, "Theory of time-resolved fluorescence under the interaction of energy transfer in a bichromophoric system: Effect of internal rotations of energy donor and acceptor", J. Chem. Phys., 109, 1084-1092 (1998)

[38] J. Enderlein, R. Erdmann, "Fast fitting of multi-exponential decay curves", Opt. Comms. 134, 371378 (1997) 